Características del liderazgo en las empresas industriales de la ciudad y provincia de Loja

Número Publicado el 15 de enero de 2018

DOI: $10.23857 /$ dc.v4i1.847

(c)

Ciencias económicas y empresariales

Artículo de investigación

\title{
Características del liderazgo en las empresas industriales de la ciudad y provincia de Loja
}

\section{Characteristics of leadership in industrial companies in the city and province of Loja}

\section{Características de liderança em empresas industriais na cidade e província de Loja}

\author{
Carmen Cevallos-Cueva ${ }^{1}$ \\ carmen.cevallos@unl.ed.ec \\ Rocío del C. Toral-Tinitana II \\ rocio.toral@unl.edu.ec \\ Manuel E. Pasaca-Mora III \\ manuel.pasaca@unl.edu.ec \\ Ney Alfredo Gallardo IV \\ ney.gallardo@unl.edu.ec
}

Recibido: 23 de septiembre de 2017 *Corregido: 19 de octubre de 2017 * Aceptado: 18 de diciembre de 2017

1. Magíster en Docencia Universitaria e Investigación Educativa, Magíster en Administración de Empresas, Diplomado Superior en Planificación de Desarrollo Local, Diploma Superior en Gestión del Talento Humano, Licenciada en Administración de Empresas, Ingeniera Comercial, Docente Titular Principal de la Universidad Nacional de Loja, Loja, Ecuador.

II. Magíster en Docencia Universitaria e Investigación Educativa, Magister en Administración de Empresas, Licenciada en Administración de Empresas, Ingeniera Comercial, Docente Titular Principal de la Universidad Nacional de Loja, Loja, Ecuador.

III. Magíster en Docencia Universitaria en el Sistema Modular, Licenciado en Administración de Empresas, Ingeniero Comercial, Docente Titular Principal de la Universidad Nacional de Loja, Loja, Ecuador.

Iv. Magíster en Docencia Universitaria en el Sistema Modular, Licenciado en Administración de Empresas, Ingeniero Comercial, Docente Titular Principal de la Universidad Nacional de Loja, Loja, Ecuador. 


\title{
Resumen
}

El estudio trata de determinar las características de los estilos de liderazgo ejercido por los gerentes de las empresas industriales de la Provincia de Loja. La medición de los estilos de liderazgo se hizo a través de una encuesta basada en la teoría de las Características de los tipos de liderazgo. Los cuestionarios fueron aplicados a 148 trabajadores de 12 empresas industriales registradas en la Cámara de Industria de Loja. Luego de haber sido sometidos a prueba de validez y confiabilidad. Igualmente, los resultados y la teoría permiten establecer que un estilo de liderazgo corresponde a un tipo específico de gerente, evidenciándose de esta manera que existe una relación intrínseca entre estilo de liderazgo y gerencia. Los estilos de liderazgo que predominan en los gerentes de las empresas industriales de Loja son: el liderazgo emotivo libre, liderazgo racional y generativo nutritivo, en orden de importancia. Los que menos se presentan son el liderazgo generativo punitivo y el emotivo indócil. Palabras clave: estilo; liderazgo; características; empresa; industria.

\begin{abstract}
The study tries to determine the characteristics of the styles of leadership exercised by the managers of the industrial companies of the Province of Loja. The measurement of leadership styles was made through a survey based on the theory of Characteristics of leadership types. The questionnaires were applied to 148 workers from 12 industrial companies registered in the Chamber of Industry of Loja. After having been tested for validity and reliability. Likewise, the results and the theory allow establishing that a leadership style corresponds to a specific type of manager, evidencing in this way that there is an intrinsic relationship between leadership style and management. The leadership styles that predominate in the managers of the industrial companies of Loja are: free emotional leadership, rational and generative nutritive leadership, in order of importance. The least presented are the punitive generative leadership and the unruly emotional.
\end{abstract}

Keywords: Style; leadership; characteristics; company; industry.

\section{Resumo}

O estudo procura determinar as características dos estilos de liderança exercidos pelos gestores das empresas industriais da Província de Loja. A medição dos estilos de liderança foi feita através de uma pesquisa baseada na teoria das características dos tipos de liderança. Os questionários foram aplicados a 148 trabalhadores de 12 empresas industriais cadastradas na Câmara de Indústria de Loja. Depois 
de ter sido testado quanto à validade e confiabilidade. Da mesma forma, os resultados e a teoria permitem estabelecer que um estilo de liderança corresponde a um tipo específico de gestor, evidenciando, dessa forma, que existe uma relação intrínseca entre estilo de liderança e gestão. Os estilos de liderança que predominam nos gestores das empresas industriais de Loja são: liderança emocional livre, liderança nutritiva racional e generativa, em ordem de importância. Os menos apresentados são a liderança generativa punitiva e o emocional indisciplinado.

Palavras chave: Estilo; liderança; características; empresa; industria.

\section{Introducción}

El estudio trata de caracterizar los estilos de liderazgo ejercidos por los gerentes en las diversas empresas industriales de la Provincia de Loja".

La medición de los estilos de liderazgo, se hizo a través de una encuesta basada en la teoría de las Características de los tipos de liderazgo. Esta teoría señala que el liderazgo implica funciones de dirección, mando y responsabilidad reflejadas en seis tipos: generativo punitivo, generativo nutritivo, racional, emotivo libre, emotivo dócil, emotivo indócil.

Los resultados presentan los antecedentes del estudio; se analizan las teorías básicas de liderazgo y definen los términos básicos utilizados en la investigación, se expone el planteamiento del estudio: se enuncia y formula el problema; Se describen los objetivos; se enuncia la justificación y viabilidad del estudio; Se plantean las hipótesis de trabajo y se describe el método de estudio, se presentan los resultados del estudio: la caracterización de los estilos de liderazgo, y la relación los estilos adoptados por los gerentes en las empresas objeto de estudio. En igual forma se presentan conclusiones, recomendaciones, consultas realizadas y anexos.

\section{Antecedentes.}

Cuadrado Isabel, Molero Fernando y Navas Marisol (2003), realizaron un estudio denominado "El liderazgo de hombres y mujeres: diferencias en estilos de liderazgo, relaciones entre estilos y predictores de variables de resultado organizacional", con el propósito de averiguar las diferencias percibidas en el estilo de liderazgo de hombres y mujeres líderes. Asimismo, conocer las diferencias entre líderes de distinto sexo en dos aspectos: a) las relaciones existentes entre los comportamientos auto percibidos referidos a estos estilos; y, b) los estilos que mejor predicen la eficacia de la unidado 
departamento, la satisfacción con distintos aspectos de su trabajo y el esfuerzo extra logrado en sus subordinados La muestra estuvo conformada por 118 personas (65 hombres y 53 mujeres), con puestos de responsabilidad. Los resultados muestran que en general, hombres y mujeres perciben que adoptan similares estilos de liderazgo, pero las relaciones entre ellos y los estilos que predicen los resultados organizacionales, son diferentes en cada caso.

Ramírez Marbelis Suarez Carlos (2007), en su ponencia "Estilos de Liderazgo y sus Enfoques Gerenciales", producto de una investigación cuyo objetivo fue explicar los estilos de liderazgo y sus enfoques gerenciales. Para ello se estudiaron un cuerpo de teorías referidas a estilos de liderazgo y gerencia, de un grupo seleccionado de autores contemporáneos. Metodológicamente la investigación fue de tipo documental, para lo cual se aplicaron reglas y procedimientos intelectuales propios del método científico. En donde se utilizó la observación documental y el análisis de documentos; el instrumento utilizado fue la ficha bibliográfica que permitieron realizar una aproximación teóricometodológica de las variables de estudio, para entender el papel que juegan los líderes y gerentes en las metas propuestas en cualquier organización. Los resultados se evidencian en el análisis de los objetivos propuestos. De esta manera estableció que un estilo de liderazgo corresponde a un tipo específico de gerente, es decir, existe una relación intrínseca entre estilo de liderazgo y gerencia.

Pedraza Norma, Verastegui Gerardo y Bernal Idolina (2014), en su estudio "Prácticas de Liderazgo en Empresas Comerciales en Tamaulipas (MÉXICO)", cuyo objetivo fue identificar las prácticas de liderazgo en empresas del sector comercial en Tamaulipas (México). En donde se aplicó un cuestionario revisado por expertos a una muestra de 84 gerentes. Se usó el análisis factorial exploratorio para identificar la estructura subyacente de las prácticas de liderazgo. Además, los datos fueron analizados con estadística descriptiva, prueba t y ANOVA. Se identificó que las cinco dimensiones del Inventario de Prácticas de Liderazgo (IPL) que están presentes en el ejercicio de liderazgo de la gerencia comercial de las empresas analizadas fueron: desafiar los procesos, inspirar una visión compartida, habilitar a los demás para actuar, modelar el camino y dar aliento al corazón. Se observó en este estudio que las cinco dimensiones están presentes en las prácticas de liderazgo de la gerencia comercial.

Pazmiño Arturo, Beltrán Mayra y Otros (2016), investigan los Estilos de Liderazgo y su influencia en el desarrollo empresarial E: Caso Pymes, provincia de Tungurahua Ecuador, el objetivo fue 
determinar el estilo de liderazgo que los administradores, utilizan para el proceso de toma de decisiones gerenciales, realizando inicialmente una aproximación teórica-metodológica respecto del fenómeno del liderazgo. Para determinar el estilo de liderazgo, se analiza en primera instancia el experimento realizado por Kurt Lewin y sus resultados para señalar los tres estilos predominantes en el quehacer administrativo: el Autocrático, el Democrático y el Laissez Faire (liberal o consultivo). La metodología utilizada se basa en la aplicación de encuestas a todas las Pymes registradas, que son 1.220 empresas dedicadas a diversas actividades industriales y de servicios a la industria, de acuerdo con los datos reportados por el Servicio de Rentas Internas del año 2013, se ubicaron las Pymes por los nueve cantones de la provincia, para luego analizar los resultados y relacionarlos con los emprendimientos del sector. Finalmente, se realizan varias reflexiones sobre los resultados considerando el predominio del estilo de liderazgo en las Pymes de la provincia y su influencia en el desarrollo empresarial.

Galarza Sandra, García Juanita y otros (2017) realizan estudios sobre la "Estructura organizacional y estilos de liderazgo en Cooperativas de Ahorro y Crédito de Pichincha" Los estudios sobre liderazgo resultan de mucho interés tanto para las organizaciones como para los científicos de la administración, por la existencia de una diversidad de teorías y modelos que se han venido perfeccionando con el paso del tiempo. El objetivo fue determinar cualitativamente los estilos de liderazgo utilizados por los gerentes de las Cooperativas de Ahorro y Crédito de la provincia Pichincha y relacionarlos con las estructuras organizacionales a través de las cuales operan estas instituciones. La metodología se basó en la aplicación de encuestas a directivos de 60 cooperativas, tomando como referencia las variables planteadas por Bass y Avolio en su "Cuestionario Multifactorial sobre Liderazgo MLQ", unido a otras metodologías para la evaluación de la estructura organizacional. Se concluye que en las cooperativas estudiadas los estilos utilizados por directivos son: transaccional, transformacional y Laissez Faire, siendo este último el que predomina en las estructuras organizacionales

La Cámara de Industrias de Loja, fue creada con base al Decreto Supremo Nro. 51, de 20 de Agosto de 1936; conformada de conformidad con la Ley, como una persona jurídica se rige es administrada por el directorio, el mismo está conformado de la siguiente manera: Presidente, Vicepresidente, tres Vocales Principales y tres Suplentes, todos elegidos por la Asamblea General Ordinaria correspondiente al mes de enero de cada año: durarán un año en el ejercicio de sus funciones y podrán ser indefinidamente reelegidos. 


\section{Marco teórico}

\section{Las Organizaciones}

Gibson, Invancevich y Donnelly, (1987), conciben a la organización como un grupo social, que se diferencia de otras organizaciones de personas porque su conducta se orienta hacia metas. Se asume que las metas y objetivos se alcanzan con más eficacia mediante la acción concertada de las personas. Coincidentemente Luhman (1998), las define como sistemas sociales, cuyo factor que une a sus miembros son los objetivos que corresponde a un componente específico. Igualmente, Rodríguez (2005), las concibe como sistemas sociales que tienen la particular característica de condicionar la pertenencia. Las organizaciones, establecen condiciones a las personas que quieran ingresar y pertenecer a ellas. Chiavenato, Idalberto (2009), las organizaciones son instituciones sociales que promueven la creatividad y el ingenio humano. Añade que la vida de las personas en la organización, se compone de una infinidad de interacciones con otros individuos y organizaciones y por sus limitaciones individuales se ven obligados a cooperar entre sí, para lograr ciertos objetivos que en forma individual o aislada no alcanzarían. Para el autor la cooperación entre éstas es esencial. Una organización existe cuando hay personas capaces de comunicarse, dispuestas a contribuir en una acción conjunta, a fin de alcanzar un objetivo común. La contribución de cada integrante varía en gran medida en función de las diferencias individuales y del sistema de recompensas de la organización.

\section{Las personas y las organizaciones}

Las personas se agrupan para formar organizaciones y por medio de ellas lograr objetivos comunes que individualmente serian imposible de alcanzar. Los objetivos compartidos son la premisa para el crecimiento y permanencia de las organizaciones. El crecimiento exige un mayor número de personas. Por tanto, un mayor número de objetivos individuales, que pueden entrar en conflicto con los organizacionales. El crecimiento genera un mayor número de interacciones ente personas y organización, complejas y dinámicas. Dicha interacción funciona como un proceso de reciprocidad basado en un contrato psicológico lleno de expectativas reciprocas; donde, por un lado, las organizaciones ofrecen incentivos o estímulos y por otro las personas ofrecen contribuciones. El equilibrio organizacional depende del intercambio entre incentivos ofrecidos y contribuciones como ganancia para la organización. 


\section{Estructura organizativa de la empresa}

El concepto de estructura organizativa ha recibido diferentes definiciones, aunque la mayoría de la literatura converge en su consideración como "red de comunicación" (Mintzberg, 1984). Las siguientes definiciones permiten clarificar el concepto y elementos que conforman la estructura organizativa de la empresa como unidad productiva. Para Bueno Et al (1991), la estructura orgánica de la empresa articula los recursos humanos a través de: a) la asignación más idónea de las tareas; b) la determinación de niveles de autoridad y responsabilidad apropiados a los diferentes niveles de decisión; y, c) comunicaciones notificadas en forma deseada, tanto en cantidad como en calidad. De manera similar Koontz y Weihrich (1991), conciben a la estructura de la organización como: a) identificar y clasificar las actividades requeridas; b) agrupar las actividades, c) asignar un gerente a cada agrupación con autoridad para supervisarlo; y, d) realizar una coordinación horizontal y vertical en la estructura. Robbins (1987), compara a la estructura de la organización con el esqueleto de los seres humanos que definen sus parámetros, igual las organizaciones tienen estructuras que definen los suyos. Simón (1964), la define como un complejo diseño de comunicaciones y demás relaciones existentes dentro de un grupo de seres humanos.

\section{El Liderazgo}

Frecuentemente encontramos a directivos eficientes para desempeñar sus funciones, pero sin habilidades para motivar. En sentido contrario, hay líderes con habilidad para desatar entusiasmo, pero sin habilidades administrativas para canalizar energía en la consecución de los objetivos organizacionales. Es pues un desafío para las empresas contar con gerentes que posean habilidades de liderazgo. El liderazgo se define como un proceso de influencia del directivo sobre sus trabajadores, para alcanzar metas de trabajo prefijadas (Hampton, 1993, pág. 469).

Similar parecer tienen Franklin Enrique y Krieger Mario, (2011), quienes amplían el concepto a nivel grupal y organizacional y lo define como la capacidad de influir en las actividades de una persona, grupo o equipo que forma parte de una empresa y para alcanzar objetivos y metas en cierta situación. Para estos autores, liderar implica influir, orientar marcar el rumbo, convencer, contener, comprender, motivar, capacitar, dar ejemplo, innovar, tener una visión de éxito. El líder se enfoca en las personas y equipos, inicia y origina procesos; se interesa por desentrañar el ¿por qué de las cosas?; es capaz de asumir riesgos, busca superar la cotidianeidad, piensa de manera estratégica, su visión es demediano 
y largo plazo; en esencia para ejercer liderazgo es necesario contar con seguidores, lo que convierte a una persona en líder es la disposición de los demás a seguirla. Para Hersey Paul y Kenneth Blanchard (1993), el líder necesita de tres habilidades: diagnosticar, es decir, entender la situación en la que se trata de influir; adaptar, modificar su comportamiento y el de los otros recursos de que dispone para enfrentar las contingencias de la situación y comunicar, es decir relacionarse con los demás de modo que entiendan y consecuentemente acepten; las tres habilidades, son factibles de ser desarrolladas, e implican entrenamientos planificados.

\section{Teorías de Liderazgo}

Luff, Josdeph (1994), citado por Dalton Merie, (2007), indica que la teoría del "gran hombre", la primera en surgir, supone que se "nace para ser líder" y se asume ese papel en el momento oportuno. Esta teoría establece que estos individuos evidencian su liderazgo, en su puesto de trabajo, en educación o por el simple contacto con líderes prominentes. Hoy se conoce que pocos líderes nacen así; la mayoría aprende a ser líder, estudiando, observando y trabajando duro. Las teorías modernas se dividen en tres grandes categorías, teoría de rasgos, teorías conductuales y teorías situacionales, las mismas que han evolucionado con el tiempo y con las investigaciones y todas ellas, de alguna manera, están ligadas a sus predecesoras.

Franklin, Enrique y Krieger Mario, (2011), realizan un análisis somero sobre las principales teorías sobre el liderazgo, con el propósito de rescatar la riqueza de los distintos enfoques, que ayudan a comprender mejor este aspecto importante en el desarrollo de grupos, equipos, organizaciones y sociedades.

\section{- Teorías de los rasgos de personalidad}

Esta teoría afirma que se nace con la personalidad, aptitudes y los rasgos necesarios para ser líder. Se ha utilizado para caracterizar a los grandes líderes de la humanidad, consiste en identificar las características de personalidad, sociales, físicas o intelectuales que diferencian a los líderes de quienes no lo son, ejemplo: ambición y energía, deseo de dirigir, honestidad e integridad, confianza en uno mismo, inteligencia, juicio, buen sentido, carácter motivador y conocimiento adecuado para el puesto. Este enfoque no prosperó, pues no hay acuerdo respecto de qué características identifican la personalidad de líder y porque muchas de éstas son reconocibles también en sus seguidores. 
La conclusión, es que el liderazgo pareciera ser más bien una condición situacional o adquirida mediante el aprendizaje, vivencias, experiencias personales y aprovechamiento de oportunidades, etc. o una conjunción de personalidad y situaciones.

De igual forma se comprobó, que en ocasiones se combinan factores de personalidad y habilidades en determinadas situaciones, ejemplo: motivación para desarrollar objetivos y metas estratégicas; confianza en sí mismo las mismas que se trasmiten a los seguidores y luego se transforma en seguridad. Otros rasgos fáciles de distinguir son capacidad de comprensión, intuición, contención emocional, facilidad para trasmitir valores, habilidad para comunicar, relacionarse y enseñar, el talento para dirigir, crear, delegar y desarrollar equipos, manejar y resolver conflictos.

\section{- Teorías del liderazgo carismático}

Max Weber (1981), define al carisma como "la cualidad que pasa por extraordinaria de una personalidad por cuya virtud, se lo considera en posesión de fuerzas sobrenaturales o sobrehumanas o por lo menos, específicamente extraordinarias y no asequibles por cualquier otro". En ese orden de pensamiento, el término carisma se refiere a la tendencia de los seguidores de hacer contribuciones extraordinarias a ciertos comportamientos que encarna el líder.

La teoría se basa en dos modelos; el del rey pastor que muestra el camino y el del rey tejedor preferido por Platón, que busca conciliar las personalidades transformadoras y conservadoras, es decir, compatible con aquello que en principio no lo es. En este modelo el líder busca proteger al grupo frente a las amenazas y vela para que no se desintegre.

El liderazgo puede ser distante o cercano, es posible que los seguidores lo amen, aun cuando él no demuestre reciprocidad o se comporte indiferente y hasta implacable. Desde su punto de vista debe tener dominio de sí mismo, para poder dominar la situación, maneja las variables tiempo, sobre el cual no admite imposiciones. Es él quien establece el marco temporal y crea su propio ritmo y espacio en donde busca la ventaja de fuerzas, en el lugar y momento de la decisión. Ejerce la diferencia metódica, a diferencia del intelectual, que se caracteriza por la duda, por la interrogante permanente. Busca la eficacia en la acción y para ello organiza a los seguidores. 


\section{- Teorías del comportamiento}

Estas teorías expresan, que, si bien ciertos comportamientos específicos diferencian a los líderes de los no líderes, las cualidades que identifica a los primeros no necesariamente son innatas; entonces, es posible capacitar a una persona para ser líder, está teoría no toma en cuenta el contexto. Existen diferentes corrientes desarrolladas en torno a estas teorías:

Kurt Lewin, habla de tres estilos de liderazgo: autocrático, liberal y democrático. El autoritario, es centralista, dominante, controlador y monopolizador. El liberal, deja hacer, es permisivo y delega. El democrático, fija objetivos, es participativo, orientador, controla resultados, descentraliza, empodera y faculta.

Blake Mouton y la teoría de la Rejilla Administrativa, se construye en base a dos dimensiones: preocupación por las personas y la producción. La tabla de doble entrada, identifica nueve posiciones. Su hipótesis es que la mejor manera de administrar se basa en los equipos de trabajo. Dadas sus características se presta para fomentar el comportamiento y la participación de los miembros, la buena comunicación, la confianza mutua y el tratamiento de los problemas. La matriz propuesta por los autores establece cuatro estilos extremos de liderazgo. El equilibrado, autocrático, administración de tipo "Club de amigos" y administración pobre.

House y Mitchell (1974), proponen la Teoría Trayectoria Meta, esta teoría sugiere que el líder es eficaz en función del impacto que tenga en la motivación y satisfacción de los seguidores. Se centra en la forma cómo el líder influye sobre la percepción que tienen sus seguidores respecto a sus metas laborales, sus objetivos personales y los caminos para alcanzarlos. Según esta teoría el líder actúa por dos vías principales: aclarando la ruta y aumentando las recompensas

\section{- Teoría del liderazgo transaccional}

Se basa en el intercambio de premios por esfuerzos, el líder se preocupa por hacer que las tareas se realicen, centrando su interés en las condiciones del momento, este tipo de liderazgo motiva y guía a sus seguidores hacia la consecución de las metas mediante la clarificación de los requerimientos de papeles y tareas. 


\section{Características de los Liderazgos}

Drucker (1989), citado en la revista de Psicología de piscología ISS 1990, 66757, expresa que los líderes eficaces son capaces de dirigir, impulsar y supervisar a sus colaboradores, así mismo, se espera que los directivos y los miembros que ocupan puestos de responsabilidad sobre otros individuos en una organización. En consecuencia, se puede afirmar que el liderazgo implica funciones de dirección, de mando y de responsabilidad; pudiendo expresarse en los siguientes tipos:

- Liderazgo Generativo Punitivo (GP): El líder preocupado en la producción, posesivo e inflexible, exigente y conservador, genera presión al grupo y toma una decisión sin anunciarla.

- El liderazgo Generativo Nutritivo (GN): El líder se caracteriza por hacer el bien al grupo, lo nutre, da libertad y es generoso. Se siente tranquilo mientras comprueba los progresos del grupo.

- Liderazgo Racional (R): El líder se caracteriza por hacer que el grupo marche en ausencia del líder, capta las necesidades del grupo, recibe ideas y sugerencias, respeta y confía en el grupo; es considerado, servicial y amistoso, en síntesis, es competente.

- Liderazgo Emotivo Libre (EL): El líder hace lo que siente, es natural, espontáneo, expresa emociones auténticas, aspira a ser líder racional, pero con cierto grado de inseguridad sobre su éxito.

- Liderazgo emotivo dócil (ED): El líder hace lo que le dicen, presenta un alto grado de sumisión, tiene vergüenza y sentimientos de culpa.

- Liderazgo emotivo indócil (EI): El líder hace lo contrario a lo que le dicen, presenta cargas de resentimiento, celos, rencor y grados de rebeldía; el grupo funciona por su presión y sólo cuando está presente el líder.

\section{Escala de Liderazgo organizacional. (ELO).}

La Escala de Liderazgo Organizacional (ELO), fue elaborada por el Equipo de Asesoría y Consultoría de Personal (1998) - Perú, con el objetivo de medir el comportamiento del líder dentro y fuera de la organización, determinando algunas características y particularidades de su accionar, dependiendo de los tipos de grupos en los cuales interactúa. Se basa en una escala de actitudes tipo Likert, donde se 
evalúan los estilos de liderazgo, este instrumento, además de brindar confiabilidad en trabajo de diagnóstico organizacional, posibilita un nivel de comparación entre estilos de liderazgo y la búsqueda de nuevos líderes.

La Escala Liderazgo Organizacional (Egoavil, s/f), permite evaluar la forma de conducción personal del grupo y cómo el líder logra establecer un clima organizacional, para ello propone seis tipos de liderazgo: Generativo Punitivo (GP); Generativo Nutritivo (N); Racional (R); Emotivo Libre (L); Emotivo Dócil (ED); Emotivo Indócil (EI). Los datos son recabados mediante un cuestionario, son sistematizados a través de procedimientos de estadística descriptiva, para luego ser analizados y determinar la incidencia de los estilos de liderazgo en el clima organizacional y el estilo predominante en la gestión de las empresas industriales de la ciudad y provincia de Loja.

\section{Materiales y métodos}

La presente investigación, se llevó a efecto a través del método inductivo, aplicable a las ciencias sociales y administrativas, en razón de que trata de determinar los por los estilos de liderazgo predominantes en las empresas industriales de la ciudad y provincia de Loja.

El diseño de investigación es no experimental de tipo transversal, en razón de que no se manipuló deliberadamente las variables en estudio; la información fue recogida tal y como se encontró en los sujetos de investigación. Es de corte transversal porque la información fue recogida en un instante del tiempo. Se trata de una investigación descriptiva; pues el estudio pretende caracterizar los estilos de liderazgo predominantes, adoptados por los gerentes de las empresas objeto de estudio.

La población la constituyo las empresas industriales de la provincia de Loja, en donde aplicó encuestas a un total de 142 empleados y funcionarios que laboran en la planta administrativa de cada una de ellas.

Los datos fueron recolectados a través de una encuesta, para cuyo efecto se elaboró un cuestionario sobre la Variable: Estilos de Liderazgo

Para cumplir los objetivos de la investigación se aplicó un cuestionario para medir la categoría de liderazgo que predomina en los directivos de las empresas. Este instrumento es una escala con valoraciones entre el 1 al 10. Si la frase caracteriza a la conducta lo más fielmente, entonces será 
valorado con 10 puntos, mientras que, si ve que no reflejan la conducta, puede calificar como 0 . En esta escala, el puntaje de 5, indica que esta conducta es manifiesta de vez en cuando, mientras que los valores intermedios Extremos (2,3 y 4 o 6,7 y 8), son conductas que se acercan o se alejan de su comportamiento promedio.

\section{Resultados}

Los resultados de la investigación se presentan a continuación

La tabla 1 da cuenta de la valoración de los aspectos relacionados al liderazgo generativo punitivo; se observa que, en relación a la actitud crítica, el promedio es de alrededor de 5 puntos, es decir que los evaluados asumen esta actitud de vez en cuando. La actitud amenazadora se presenta muy pocas veces, según lo evidencia el promedio de 2 puntos; algo similar sucede con la actitud autoritaria, que también se manifiesta muy poco; el promedio en este caso está cerca a los 4 puntos. En cambio, la actitud severa y exigente, con un promedio de 6 puntos, se observa que se manifiesta con bastante frecuencia.

Tabla 1. Valoración de los elementos del liderazgo generativo punitivo

\begin{tabular}{|l|c|c|}
\hline ELEMENTOS & PROMEDIO & $\begin{array}{c}\text { DESVIACIÓN } \\
\text { ESTÁNDAR }\end{array}$ \\
\hline 1 ¿Tu jefe critica lo que te rodea? & 5.1 & 2.5 \\
\hline 7 ¿Tu jefe le gusta amenazar? & 2.2 & 2.0 \\
\hline 13 ¿Tu jefe es severo y exigente? & 6.3 & 2.3 \\
\hline 19 ¿Tu jefe es autoritario? & 3.9 & 2.6 \\
\hline
\end{tabular}

Fuente: Cuestionario de Estilos de Liderazgo Organizacional (ELO)

En cuanto a los elementos de liderazgo generativo nutritivo tabla 2, los promedios se encuentran entre 6 y 8 puntos, lo que significa que los investigados manifiestan bastante preocupación por los demás, brindan consejos y orientación con mucha frecuencia, están dispuestos a motivar e incentivar, así como a proteger y orientar.

Tabla 2. Valoración de los elementos del liderazgo generativo nutritivo

\begin{tabular}{|l|c|c|}
\hline \multicolumn{1}{|c|}{ ELEMENTOS } & Promedio & Desviación estándar \\
\hline 2 iTu jefe se preocupa por los demás? & 6.7 & 2.4 \\
\hline 8 iTu jefe le agrada aconsejar y orientar? & 7.7 & 1.7 \\
\hline 14 iTu jefe le gusta motivar e incentivar? & 7.9 & 2.0 \\
\hline
\end{tabular}


Características del liderazgo en las empresas industriales de la ciudad y provincia de Loja

\begin{tabular}{|l|l|l|}
\hline 20 ¿Tu jefe le agrada proteger y orientar? & 7.7 & 1.7 \\
\hline
\end{tabular}

Fuente: Cuestionario de Estilos de Liderazgo Organizacional (ELO)

Elaboración: Los autores.

El estudio también revela que los elementos del liderazgo racional tabla 3, se presentan con bastante frecuencia; hay una actitud de confrontación con la realidad, los directivos están bastante dispuestos para el análisis y la orientación, tienen una actitud positiva para informar y para aprender.

Tabla 3. Valoración de los elementos del liderazgo racional.

\begin{tabular}{|l|c|c|}
\hline ELEMENTOS & Promedio & Desviación estándar \\
\hline 3 ¿Tu jefe confronta con la realidad? & 7.8 & 2.0 \\
\hline 9 ¿Tu jefe le gusta analizar y orientar? & 7.8 & 1.9 \\
\hline 15 ¿Tu jefe solicita y brinda información? & 8.1 & 2.1 \\
\hline 21 ¿Tu jefe tomas interés en aprender? & 8.5 & 1.8 \\
\hline
\end{tabular}

Fuente: Cuestionario de Estilos de Liderazgo Organizacional (ELO)

Elaboración: Los autores.

En cuanto a los elementos del liderazgo emotivo libre tabla 4, también hay actitudes que se dan con bastante frecuencia; los promedios superan los 8 puntos. Los trabadores frecuentemente se comportan en forma natural, son entusiastas, vivaces e intuitivos y tienen un estado de ánimo optimista, que les permite estar siempre alegres.

Tabla 4. Valoración de los elementos del liderazgo emotivo libre

\begin{tabular}{|l|c|c|}
\hline ELEMENTOS & PROMEDIO & $\begin{array}{c}\text { DESVIACIÓN } \\
\text { ESTÁNDAR }\end{array}$ \\
\hline 4 ¿Tu jefe actúa en forma natural? & 8.2 & 2.3 \\
\hline 10 ¿Tu jefe le agrada ser entusiasta? & 8.3 & 2.0 \\
\hline 16 ¿Tu jefe es vivaz e intuitivo? & 8.0 & 1.9 \\
\hline 22 ¿Tu jefe le gusta estar alegre? & 8.6 & 1.6 \\
\hline
\end{tabular}

Fuente: Cuestionario de Estilos de Liderazgo Organizacional (ELO)

En cuanto al liderazgo emotivo indócil tabla 5, las actitudes hacia los aspectos que lo componen son poco frecuentes, según se desprende de los promedios inferiores a 5; es decir, los encuestados casi no originan conflictos, no sienten envidia por los demás, no les gusta culpar a las personas y no muestran muchas actitudes de resentimiento. 
Tabla 5. Valoración de los elementos del liderazgo emotivo indócil

\begin{tabular}{|l|c|c|}
\hline ELEMENTOS & PROMEDIO & $\begin{array}{c}\text { DESVIACIÓN } \\
\text { ESTÁNDAR }\end{array}$ \\
\hline 5 ¿Tu jefe origina conflictos sin querer? & 3.0 & 2.2 \\
\hline 11 ¿Tu jefe siente envidia por los demás? & 2.9 & 2.5 \\
\hline 17 ¿Tu jefe le gusta culpar a las personas? & 2.7 & 2.5 \\
\hline 23 ¿Tu jefe es resentido? & 4.3 & 3.0 \\
\hline
\end{tabular}

Fuente: Cuestionario de Estilos de Liderazgo Organizacional (ELO)

Elaboración: Los autores.

Con respecto al liderazgo emotivo dócil tabla 6 , los resultados muestran que son pocos los encuestados que muestran una actitud nerviosa y son inseguros, según se observan en los promedios cercanos a los 4 puntos; en cambio, si se observa que hay una actitud colaborativa y de predisposición a aceptar lo que otros dicen y hacen.

Tabla 6. Valoración de los elementos del liderazgo emotivo dócil

\begin{tabular}{|l|c|c|}
\hline ELEMENTOS & PROMEDIO & $\begin{array}{c}\text { DESVIACIÓN } \\
\text { ESTÁNDAR }\end{array}$ \\
\hline 6 ¿Tu jefe se siente con actitud nerviosa? & 3.9 & 2.9 \\
\hline 12 ¿Tu jefe le gusta orientar a otros? & 7.9 & 2.0 \\
\hline 18 ¿Tu jefe se siente inseguro? & 3.7 & 3.0 \\
\hline 24 ¿Tu jefe acepta todo de los demás? & 5.7 & 2.3 \\
\hline
\end{tabular}

Fuente: Cuestionario de Estilos de Liderazgo Organizacional (ELO)

Elaboración: Los autores.

Los datos de la tabla 7 dan cuenta que los tipos de liderazgo que más perciben los encuestados, son el liderazgo generativo nutritivo, liderazgo racional y liderazgo emotivo libre; en estas tres categorías, el $79.4 \%$, 85.9\% y 87\%, presentan niveles con tendencia alta o niveles altos. Por el contrario, el liderazgo que menos se manifiesta en este grupo de investigados es el generativo punitivo y el emotivo indócil; el $51.1 \%$ y $69.5 \%$, de encuestados evidencia un nivel bajo o tendencia bajo. 
Características del liderazgo en las empresas industriales de la ciudad y provincia de Loja

Tabla 7. Tipos de liderazgo ejercidos por los gerentes de las empresas industriales de la ciudad de Loja

\begin{tabular}{|l|c|c|c|c|c|c|c|c|c|c|}
\hline \multirow{2}{*}{ CATEGORIAS } & \multicolumn{2}{|c|}{ BAJO } & \multicolumn{2}{|c|}{$\begin{array}{c}\text { TENDENCIA } \\
\text { BAJO }\end{array}$} & \multicolumn{2}{c|}{ PROMEDIO } & \multicolumn{2}{c|}{$\begin{array}{c}\text { TENDENCIA } \\
\text { ALTO }\end{array}$} & \multicolumn{2}{|c|}{ ALTO } \\
\cline { 2 - 11 } & $\mathbf{N}^{\mathbf{0}}$ & $\mathbf{\%}$ & $\mathbf{N}^{\mathbf{0}}$ & $\mathbf{\%}$ & $\mathbf{N}^{\mathbf{0}}$ & $\mathbf{\%}$ & $\mathbf{N}^{\mathbf{0}}$ & $\%$ & $\mathbf{N}^{\mathbf{0}}$ & $\%$ \\
\hline $\begin{array}{l}\text { Generativo } \\
\text { Punitivo }\end{array}$ & 9 & $9.8 \%$ & 38 & $41.3 \%$ & 37 & $40.2 \%$ & 5 & $5.4 \%$ & 3 & $3.3 \%$ \\
\hline $\begin{array}{l}\text { Generativo } \\
\text { Nutritivo }\end{array}$ & 1 & $1.1 \%$ & 3 & $3.3 \%$ & 15 & $16.3 \%$ & 40 & $43.5 \%$ & 33 & $35.9 \%$ \\
\hline Racional & 1 & $1.1 \%$ & 2 & $2.2 \%$ & 10 & $10.9 \%$ & 22 & $23.9 \%$ & 57 & $62.0 \%$ \\
\hline Emotivo Libre & 2 & $2.2 \%$ & 0 & $.0 \%$ & 10 & $10.9 \%$ & 23 & $25.0 \%$ & 57 & $62.0 \%$ \\
\hline Emotivo Indócil & 35 & $38.0 \%$ & 29 & $31.5 \%$ & 25 & $27.2 \%$ & 2 & $2.2 \%$ & 1 & $1.1 \%$ \\
\hline Emotivo Dócil & 2 & $2.2 \%$ & 22 & $23.9 \%$ & 42 & $45.7 \%$ & 20 & $21.7 \%$ & 6 & $6.5 \%$ \\
\hline
\end{tabular}

Fuente: Cuestionario de Estilos de Liderazgo Organizacional (ELO)

Elaboración: Los autores.

\section{Discusión}

Los resultados de la tabla 8 , ponen en evidencia desde otra perspectiva, la categoría de liderazgo que más predomina en los investigados; los promedios más altos, lo ostentan el liderazgo emotivo libre, liderazgo racional y generativo nutritivo, en ese orden de importancia; los que menos se presentan son el liderazgo generativo punitivo y el emotivo indócil; en el caso del liderazgo emotivo dócil, los resultados indican que éste se presenta de vez en cuando, según su promedio de alrededor de 5 puntos.

Tabla 8. Valoración promedio por las diferentes categorías de liderazgo

\begin{tabular}{|l|c|c|}
\hline CATEGORIA & Promedio & Desviación estándar \\
\hline Generativo Punitivo & 4.44 & 1.56 \\
\hline Generativo Nutritivo & 7.49 & 1.46 \\
\hline Racional & 8.04 & 1.54 \\
\hline Emotivo Libre & 8.23 & 1.67 \\
\hline Emotivo Indócil & 3.28 & 1.85 \\
\hline Emotivo Dócil & 5.37 & 1.73 \\
\hline
\end{tabular}

Fuente: Cuestionario de Estilos de Liderazgo Organizacional (ELO)

Elaboración: Los autores. 
Igualmente, los resultados y la teoría permiten establecer que un estilo de liderazgo corresponde a un tipo específico de gerente, evidenciándose de esta manera que existe una relación intrínseca entre estilo de liderazgo y gerencia.

Los estilos de liderazgo que predominan en los gerentes de las empresas industriales de Loja son: el liderazgo emotivo libre, liderazgo racional y generativo nutritivo, en orden de importancia. Los que menos se presentan son el liderazgo generativo punitivo y el emotivo indócil.

\section{Conclusiones}

Los estilos de liderazgo que predominan en las empresas industriales de Loja, son: el liderazgo emotivo libre, liderazgo racional y generativo nutritivo, en ese orden de importancia. Los que menos se presentan son el liderazgo generativo punitivo y el emotivo indócil.

Existe una relación significativa $(|\mathrm{r}|>0.3$,Sig. $<0.05)$, entre el liderazgo generativo nutritivo, liderazgo racional, liderazgo emotivo libre y liderazgo emotivo indócil.

\section{Referencias Bibliográficas}

CHIAVENATO, Idalberto, 2007, Las Personas y las Organizaciones en Administración de Recursos Humanos, Editorial Mc Graaw.Hill Interamericana. México

CHIAVENATO, Idalberto, 2009, Concepto de Organización en Administración de Recursos Humanos, Editorial MaGraaw. Interamericana. México

DALTON Maire y otros (2007), Relaciones Humanas. Cengage Learing editores, S.A. Tercera edición.

DALY,J. A.; FALCIONE, R. L. Y DAMHORST, M. (1979), "Communication correlates of relational and organizational satisfaction: An audit based investigation". Presentado en Annual Convention of the Intern. Communication Association Philadelphia.

DENISON, D. (1991). Cultura corporativa y productividad organizacional. Colombia: Legis.

DRUCKER (1989) citado en la revista de Psicología ISS 1990, 66757 Psicológica Estilos del Pensamiento, Titos de Liderazgo y Estilos Educativos en docentes Universitarios. 
FRANKLIN Enrique y KRIEGER Mario, 2011, Comportamiento Organizacional, Enfoque para America Latina, Editorial Person. Educación de México, S.A. de C.V.-

GUILLEN. C. Y BOZAL R (2001). Psicología del Trabajo para la Relaciones Laborarles PÁG. 169172. Ed. Nomos S.A. Colombia.

HOUSE, R. y MITCHELL, T. (1974). Path goal theory of Leadership. En J.M. Pennings (Ed.), Decision Making: an organizational behavior approach (pp. 81-97

HERSEY, Paul \& Blanchard Kenneth. (1993), Managment of Organizational Behavior. Utilizing Human Resources. (Sixth edition). Ed. Prentice Hall. New Jersey.

LITWIN,G. H. Y STRINGER, R. A. (1968), Motivation and organizational climate. Harvard Univ. Graduate School of Bussines Administration

MALDONADO, B. María (2007). Auditoría de Gestión como Herramienta para Evaluar los Procesos Administrativos, Financieros y Operativos de las Empresas Industriales Grandes.

RODRÍGUEZ M, Darío, (2005), Diagnóstico Organizacional, Editorial Alfaomega, 6ta edición, Buenos Aires.

TAYLOR, C.W. y ELLINSON, R.L. (1975), "Moving toward working models in creativity: Utah creativity experiences an insights". En I. A. TAYLOR y J.W.GETZELS (Eds.). Perspectives in creativity. Chicago: Aldine

WEBER Max. 1981, Economía y Sociedad, Fondo de Cultura Económica. México.

http://www.espol.edu.ec/alumni/200219426/index.aspx

http://www.educadormarista.com/proyectoaprender/clima-organizacional.htm,

http://sisbib.unmsm.edu.pe/bvrevistas/investigacion_psicologia/v13_n1/pdf/a10.pdf

http://www.espol.edu.ec/alumni/200219426/index.aspx

Liderazgo en las organizaciones http://www.itescam.edu.mx/principal/sylabus/fpdb/recursos/r77954.PDF 